\title{
Esclerosis múltiple en pediatría. Presentación de dos casos
}

\section{Multiple sclerosis in pediatrics. Presentation of two cases}

\author{
Julio César Martínez-Padilla,* María de Lourdes Astorga-Chávez, ${ }^{\ddagger}$ \\ María Guadalupe Mandujano-Trejo ${ }^{\ddagger}$
}

* Clínica Cuauhtémoc del Servicio de Transporte Colectivo Metro; ${ }^{\ddagger}$ Sanatorio Durango. Ciudad de México, México.

\section{RESUMEN}

Objetivo: Describir dos pacientes adolescentes con esclerosis múltiple, la cual es una enfermedad desmielinizante del sistema nervioso central. Presentación de los casos: El primer caso se trató de una paciente de 14 años con sobrepeso, con un padecimiento caracterizado por mareo, cefalea, parestesias en miembros inferiores, diplopía y bradilalia. A la exploración, datos compatibles de afectación a nivel cerebeloso. En estudio de imagen de resonancia magnética (IRM) de cráneo se evidencia áreas de desmielinización en fosa posterior. Se diagnostica esclerosis múltiple de variedad remitente recurrente. Con la administración de esteroides e interferón beta hubo mejoría de sintomatología. Segundo caso: femenino de 13 años de edad, quien presenta caída de propia altura, incontinencia de esfínter vesical, pérdida de fuerza en brazo y pierna izquierdas. En la exploración física, hemiparesia izquierda. IRM: datos de desmielinización en ventrículo lateral, cerebelo y médula espinal. La paciente mejoró al iniciar esteroides e interferón. Conclusiones: La presentación clínica de la esclerosis múltiple en pacientes pediátricos es un reto, ya que es un padecimiento que tienen manifestaciones poco específicas. Para establecer el diagnóstico se requiere realizar múltiples estudios. El pronóstico puede ser más favorable entre más temprano se inicie el tratamiento.

Palabras clave: Esclerosis múltiple, enfermedad desmielinizante, alteraciones neurológicas, resonancia magnética, adolescentes.

\begin{abstract}
Objective: We describe two adolescent patients with multiple sclerosis, which is a demyelinating disease of the central nervous system. Presentation of the cases: The first case was a 14-year-old female patient with overweight; her condition was characterized by dizziness, headache, paresthesia in the lower limbs, diplopia and bradylalia. On physical examination, compatible data of cerebellar involvement were identified. A magnetic resonance imaging (MRI) of the skull showed areas of demyelination in the posterior fossa. Multiple sclerosis, the relapsing-remitting variety, is diagnosed. After the administration of steroids and beta interferon, there was improvement in symptoms. Second case: 13-year-old female, who presented fall from her own height, bladder incontinence, loss of strength in the left arm and leg. On physical examination, left hemiparesis was detected. MRI: demyelination data in lateral ventricle, cerebellum and spinal cord. The patient improved after starting steroids and interferon. Conclusions: The clinical presentation of multiple sclerosis in pediatric patients is a challenge, since it is a condition with unspecific manifestations. Establishing the diagnosis requires multiple studies. The earlier treatment is started, the more favorable the prognosis.
\end{abstract}

Keywords: Multiple sclerosis, demyelinating disease, neurological manifestations, magnetic resonance, adolescents.

Correspondencia: Julio César Martínez-Padilla, E-mail: jucemarpa@hotmail.com

Citar como: Martínez-Padilla JC, Astorga-Chávez ML, Mandujano-Trejo MG. Esclerosis múltiple en pediatría. Presentación de dos casos. Rev Mex Pediatr. 2021; 88(3): 101-107. https://dx.doi.org/10.35366/102190 


\section{INTRODUCCIÓN}

La esclerosis múltiple es una enfermedad desmielinizante crónica que afecta al sistema nervioso central (SNC). Se caracteriza por la tríada de inflamación, desmielinización y gliosis, y constituye una causa importante de discapacidad no traumática en adultos jóvenes. Fue descrita por Jean-Martin Charcot, en el año $1868 .^{1}$

Con base en el curso clínico, se clasifica en los siguientes subtipos: remitente-recurrente, secundariamente progresiva, primariamente progresiva, progresiva recurrente. El subtipo remitente-recurrente es el más frecuente, hasta en $85 \%$ de los casos. ${ }^{2}$

Es un padecimiento poco frecuente en pediatría. Del total de casos, alrededor del 3 al 5\% ocurre en menores de 15 años. Es más común en el sexo femenino, en una relación de 3:1.3,4 Los factores de riesgo asociados al desarrollo de esclerosis múltiple son: infecciones (predominantemente virales), sexo femenino, deficiencia de vitamina $\mathrm{D}$, tabaquismo, antecedentes familiares. ${ }^{5}$ La etiología es desconocida, pero se involucran causas infecciosas (herpes virus) y causas autoinmunes. ${ }^{6}$

Para el diagnóstico se han utilizado los criterios de McDonald, los cuales han presentado diversas modificaciones, la más reciente es del año 2017 (Tabla 1).7 Dado que existen otras enfermedades con síntomas similares, es necesario un abordaje sistemático para confirmar el diagnóstico, ${ }^{8}$ como el estudio de líquido cefalorraquídeo (LCR), en específico las bandas oligoclonales. ${ }^{9,10}$ Mientras que la resonancia magnética (IRM) permanece como el método más efectivo para identificar lesiones desmielinizantes en el SNC, pues define sus características anatómicas y el número de lesiones. Los potenciales auditivos y visuales también constituyen apoyo. ${ }^{11,12}$

La terapia puede ser de tres tipos: tratamiento modificador, tratamiento del brote y sintomático. El primero tiene como objetivo reducir la frecuencia y severidad de los brotes, disminuyendo la probabilidad de formación de nuevas lesiones del cerebro y la médula espinal. La atención al brote acelera la reducción de los síntomas después de la recaída, pero no modifica la evolución de la enfermedad. Con el sintomático se trata de disminuir los síntomas y mejorar la calidad de vida. ${ }^{13}$

En México hay pocos casos reportados de esclerosis múltiple en la edad pediátrica, por lo que presentamos dos casos de pacientes adolescentes.

\section{PRESENTACIÓN DE LOS CASOS}

\section{Primer caso}

Paciente femenino de 14 años y tres meses, originaria de la Ciudad de México y residente del Estado de México. Sobrepeso (IMC $25.1 \mathrm{~kg} / \mathrm{m}^{2}$ ). Esquema de vacu-

Tabla 1: Criterios de McDonald 2017 para el diagnóstico de esclerosis múltiple.

\begin{tabular}{|c|c|c|}
\hline Presentación clínica & $\begin{array}{l}\text { Número de lesiones con evidencia } \\
\text { clínica objetiva }\end{array}$ & $\begin{array}{l}\text { Hallazgos adicionales necesarios para el diagnóstico de múltiples } \\
\text { esclerosis }\end{array}$ \\
\hline$\geq 2$ brotes clínicos & $\geq 2$ & Ninguno* \\
\hline$\geq 2$ brotes clínicos & $\begin{array}{l}1 \text { (así como evidencia clara histórica de } \\
\text { un anterior ataque con una lesión en una } \\
\text { posición anatómica distante) }\end{array}$ & Ninguno* \\
\hline$\geq 2$ & 1 & $\begin{array}{l}\text { Diseminación en el espacio debe demostrarse mediante un ataque } \\
\text { clínico adicional implicando un área diferente del SNC o mediante IRM }\end{array}$ \\
\hline Un ataque clínico & $\geq 2$ & $\begin{array}{l}\text { Diseminación en tiempo debe demostrarse mediante un ataque } \\
\text { clínico adicional o mediante IRM o demostración de bandas } \\
\text { oligoclonales específicas en LCR }\end{array}$ \\
\hline Un ataque clínico & 1 & $\begin{array}{l}\text { Diseminación en el espacio debe demostrarse mediante un ataque } \\
\text { clínico adicional implicando un área diferente del SNC o mediante } \\
\text { IRM y diseminación en tiempo debe demostrarse mediante un } \\
\text { ataque clínico adicional o mediante IRM o demostración de bandas } \\
\text { oligoclonales específicas en LCR }\end{array}$ \\
\hline
\end{tabular}

SNC = sistema nervioso central, IRM = imagen de resonancia magnética, LCR = líquido cefalorraquídeo. * Se debe realizar LCR e IRM en casos de duda. Cuando ambos son negativos, se debe ampliar la búsqueda a diagnósticos distintos. 


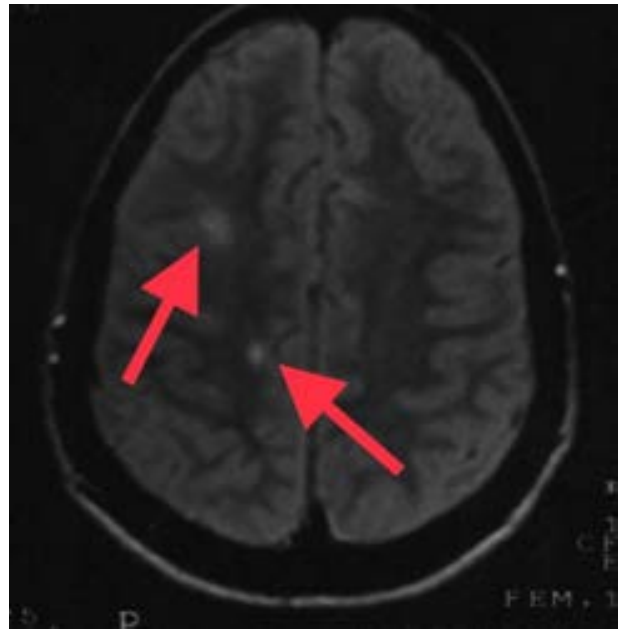

Figura 1: Resonancia magnética de cráneo secuencia en T2. Imagen axial en donde se puede observar una lesión ovoidea hiperintensa yuxtacortical (flechas).

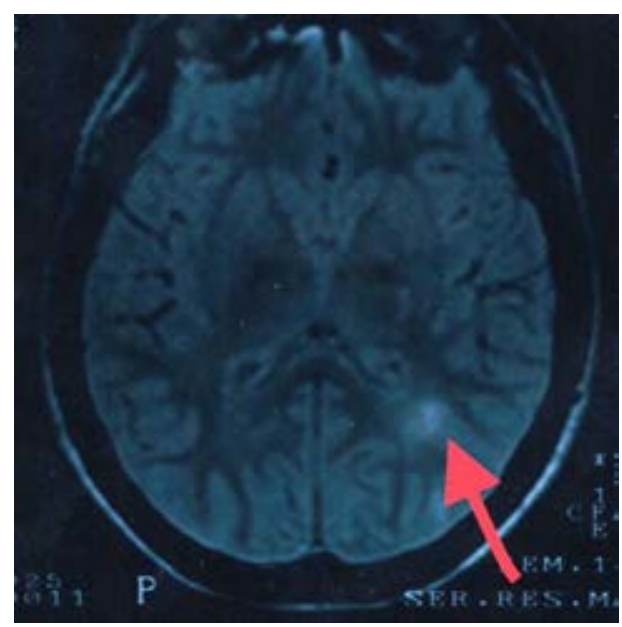

Figura 2: Resonancia magnética de cráneo secuencia en T2. Imagen axial en donde es posible observar dos lesiones ovoideas yuxtacorticales (flecha).

nación completa para la edad, sin referir vacunación reciente.

Inicia su padecimiento 30 días previos a valoración, con sensación de mareo y vértigo intermitente, agravado a la deambulación y disminuido al decúbito, en la última semana fue diario. Diez días después se agregó cefalea temporo-occipital, punzante, irradiada a región frontal, intermitente, que remitía con paracetamol. En la misma fecha se añade parestesias en miembros inferiores y, tres días previos al ingre- so, diplopía, visión borrosa, bradilalia y lenguaje escandido.

A la exploración física (EF): consciente, cooperadora, pero con marcha atáxica, lateropulsión indistinta, dismetría de predominio izquierdo, nistagmo vertical y horizontal. Se decide su ingreso hospitalario. Se solicitó biometría hemática: hemoglobina $15 \mathrm{~g} / \mathrm{L}$, hematocrito 43.8, leucocitos $8,500 \mathrm{cel} / \mathrm{mL}$, neutrófilos $63 \%$, linfocitos $30 \%$, monocitos $6 \%$, plaquetas 320,000. Tomografía computarizada de cráneo (TCC) normal. Las pruebas vestibulares, electronistagmografía y pruebas térmicas con datos indicativos de disfunción vestibular central, con componente periférico. LCR: cloruros $120 \mathrm{nmol} / \mathrm{L}$, proteínas 28 $\mathrm{mg} / \mathrm{dL}, 83$ células: leucocitos (50\%), mononucleares (40\%), polimorfonucleares (10\%), eritrocitos negativos. IgG $5.3 \mathrm{mg} / \mathrm{dL}$. Cultivo sin desarrollo. Bandas oligoclonales, policlonales y monoclonal, no detectables. Potenciales evocados auditivos: defecto de la vía auditiva rostral a la porción inferior del puente. Potenciales visuales: defecto en la conducción de la vía visual bilateral. IRM de cráneo con áreas de desmielinización (Figuras 1 a 4).

El diagnóstico de esclerosis múltiple se realizó con base en los criterios de McDonald (Tabla 1): un ataque, evidencia clínica objetiva de dos o más lesiones desmielinizantes en IRM, y hallazgos anormales en potenciales visuales y auditivos.

Se inició tratamiento con metilprednisolona, $1 \mathrm{~g}$ cada $24 \mathrm{~h}$, por cinco días, además de interferón beta1a, 6’000,000 UI. Días después se egresó por mejoría

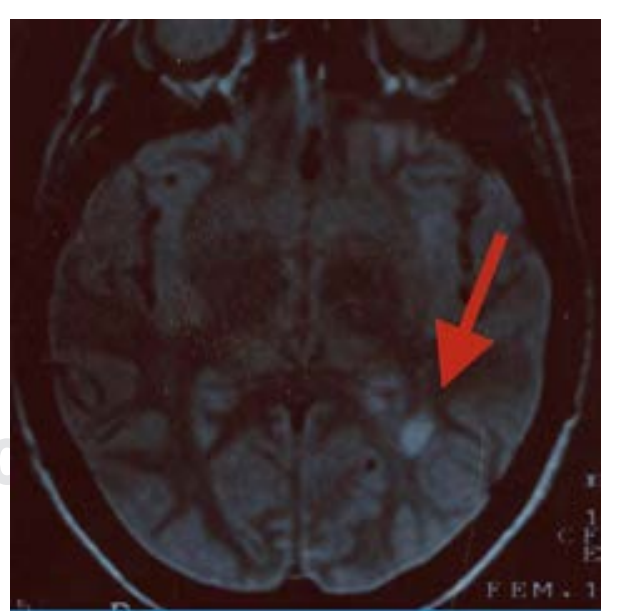

Figura 3: Resonancia magnética de cráneo secuencia en T2. Imagen coronal en donde es viable observar lesiones hiperintensas, ovoideas yuxtacorticales (flecha). 


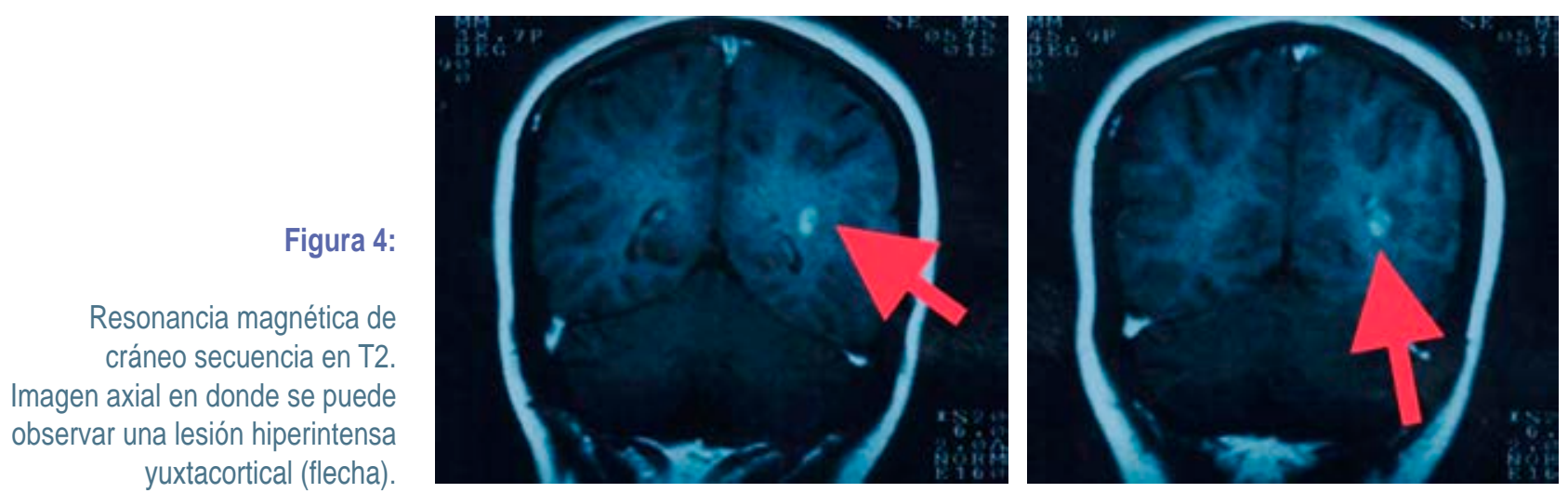

clínica, al remitir cefalea y las hipoestesias en extremidades. El lenguaje se encontraba fluido sin separación evidente de las sílabas. Persistía el nistagmo horizontal y marcha atáxica pero en menor intensidad.

A la edad de 15 años, en un nuevo evento presenta cefalea, salivación profusa, diplopía, debilidad generalizada, así como parestesias en miembros inferiores. Se da tratamiento con esteroides, acetato de glatirámero (20 mg subcutáneo cada 24 h) y terapia de rehabilitación, con lo cual hay mejoría importante en tono y fuerza de extremidades. No se reportan nuevos eventos hasta los 21 años, el cual fue de las mismas características al evento anterior. Al parecer, este último, se relacionó a la suspensión voluntaria de medicamentos.

\section{Segundo caso}

Paciente femenino de 13 años de edad, originaria y residente de Ciudad de México, con obesidad (IMC $31 \mathrm{~kg} / \mathrm{m}^{2}$ ). Esquema de vacunación completa dada la edad, no refiere vacunación reciente. Inicia su padecimiento 36 horas previo a valoración, al presentar caída de propia altura al subir escaleras, así como incontinencia vesical. Se agregó pérdida gradual en fuerza de brazo izquierdo y extremidad inferior ipsilateral, así como dolor en la misma área, hasta impedir bipedestación y deambulación. Se decide su ingreso hospitalario.

EF: hemiparesia izquierda, de predominio torácico. Tono muscular en miembros torácicos y pélvicos 2/5, hiperreflexia. Estudios de ingreso: biometría hemática con hemoglobina $15.1 \mathrm{~g} / \mathrm{L}$, hematocrito 39.8 , leucocitos $7,300 \mathrm{cel} / \mathrm{mL}$, monocitos $11 \%$, linfocitos $36 \%$, eosinófilos $2 \%$, neutrófilos $51 \%$, plaquetas 250,000, glucosa $98 \mathrm{mg} / \mathrm{dL}$, colesterol $146 \mathrm{mg} / \mathrm{dL}$, tiempos de coagulación y electrólitos séricos norma- les. En TCC: hipodensidad a nivel del brazo posterior de cápsula interna derecha y subcortical en la región parietal, sugestiva de infarto isquémico en territorio de la arteria cerebral media derecha. En ecocardiograma: corazón estructuralmente sano. Anticuerpos anticardiolipina, lúpico, anti-DNA y antinucleares negativos. Serología negativa para toxoplasmosis, rubéola, citomegalovirus y hepatitis.

Se practica una angiorresonancia, sin observarse algún trastorno vascular. IRM de cráneo y columna cervical, con áreas de desmielinización (Figuras 5 a 7). Potenciales visuales con defecto en la conducción de ojo izquierdo, y en potenciales auditivos de tallo con alteración bilateral.

LCR: glucosa $59 \mathrm{mg} / \mathrm{dL}$, proteínas $20 \mathrm{mg} / \mathrm{dL}$, pH 7.6, cloruros $118 \mathrm{nmol} / \mathrm{L}$; células: 1 y eritrocitos negativos. Bandas oligoclonales, monoclonales y policlonales no detectables, proteínas básicas de mielina $2.5 \mathrm{ng} / \mathrm{dL}$.

El diagnóstico de esclerosis múltiple se estableció con base en los criterios de McDonald: síndrome clínico aislado, alteraciones en potenciales evocados auditivos y visuales, y hallazgos de IRM de dos o más lesiones desmielinizantes (Tabla 1). Se inició tratamiento con metilprednisolona $1 \mathrm{~g}$ cada $24 \mathrm{~h}$, por cinco días, así como terapia física. Al tercer día hay mejoría, al desaparecer la incontinencia vesical, cese de hiperreflexia y mejoría del tono muscular en cuatro extremidades. Se egresa al terminar quinta dosis de metilprednisolona, posteriormente se indica interferón beta-1a, 6’000,000 UI semanal, de manera ambulatoria.

Un nuevo episodio de esclerosis múltiple ocurrió dos meses después, caracterizado por hemiparesia izquierda que requirió hospitalización. Se inició nuevo ciclo de cinco días con metilprednisolona. A los 21 años de edad se reporta un nuevo evento de características similares, por lo que se administró tratamiento a base 
de esteroides. En la actualidad, continúa manejo con interferón beta, sin nuevos eventos.

\section{DISCUSIÓN}

La esclerosis múltiple es un padecimiento considerado predominantemente de la etapa adulta, sin embargo, puede ocurrir en la edad pediátrica. El 10\% de adultos con esta enfermedad tuvieron su primera manifestación clínica antes de los 18 años. ${ }^{13}$

En México, en reportes previos, se señala que la esclerosis múltiple ocurre más frecuentemente

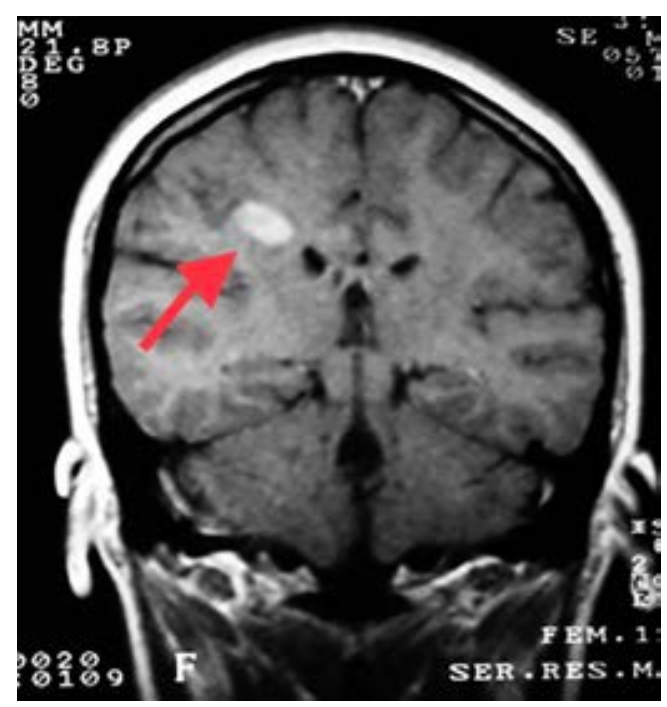

Figura 5: Resonancia magnética de cráneo secuencia en T2. Imagen coronal en donde es posible observar una lesión desmielinizante yuxtacortical (flecha). en mujeres. ${ }^{14,15}$ En concordancia, en el presente documento describimos dos pacientes adolescentes femeninos, quienes tuvieron un cuadro clínico caracterizado por múltiples síntomas y signos que hicieron difícil llegar al diagnóstico. El cuadro clínico presentado incluyó: afección ocular (descrito en 14-35\% como síntoma inicial en niños), motora, sensitiva y síndrome cerebeloso, por lo que en el abordaje se buscaron otras causas, como infecciones por citomegalovirus o virus Epstein Barr, vasculopatías, neoplasias, intoxicaciones, además de otras enfermedades autoinmunes (Tabla 2). ${ }^{16}$

En los dos casos reportados fueron de mucha utilidad los potenciales evocados, ya que ambas presentaron anormalidades en dichos estudios y permitieron, además, determinar las vías afectadas correspondientes al área de lesión. En pacientes con esclerosis múltiple se encuentran alteraciones hasta de $95 \%$ de los casos. ${ }^{17}$

La IRM sigue siendo pilar para establecer el diagnóstico. Las lesiones de la esclerosis múltiple afectan la sustancia blanca periventricular predominantemente, pero también cuerpo calloso, lóbulos temporales, tallo cerebral, cerebelo y médula espinal. Estas lesiones suelen ser redondeadas o de forma ovoide, con sentido perpendicular al sistema ventricular. Mientras que las lesiones en médula espinal son habitualmente laterales o posteriores, sin incluir más de dos cuerpos vertebrales. ${ }^{17}$ En los dos casos que presentamos hubo lesiones desmielinizantes en IRM de cráneo y, en el segundo caso, una lesión a nivel cervical.

La importancia de llegar más rápido al diagnóstico es para iniciar lo más pronto posible el tratamiento

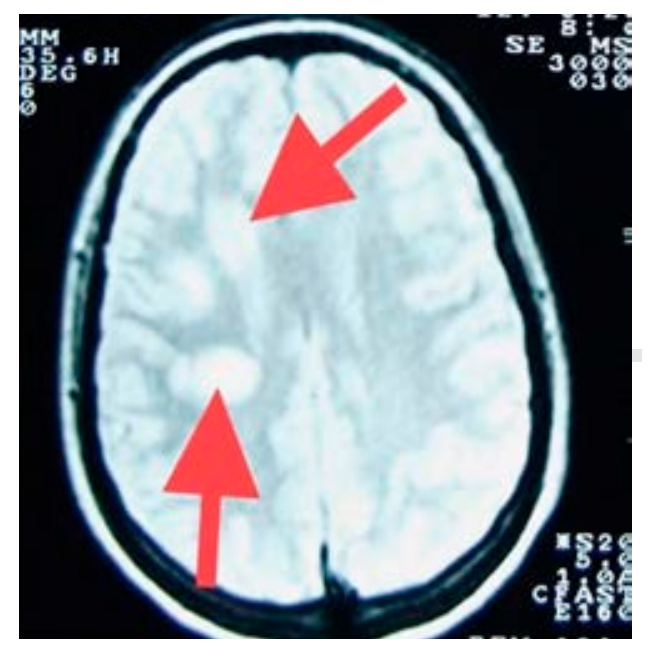

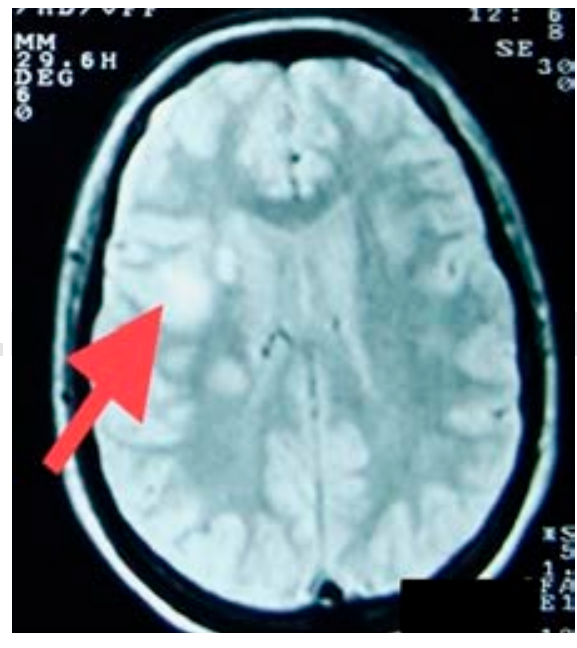

Figura 6:

Resonancia magnética de cráneo secuencia en T2. Imágenes axiales en donde es posible observar lesiones hiperintensas, ovoideas yuxtacorticales y parenquimatosas (flechas). 
y evitar las discapacidades. Sin embargo, no existe algún tratamiento que modifique los efectos sobre las lesiones ya establecidas. El daño neurológico causado por las áreas de desmielinización ocasiona afectación de la calidad de vida, según la magnitud del daño. Estos pacientes requieren manejo multidisciplinario,

Figura 7:

Resonancia magnética de médula espinal corte sagital en T2, se observa placa desmielinizante medular en el nivel de C3-C4 de aproximadamente $10 \mathrm{~mm}$ de diámetro (flecha).

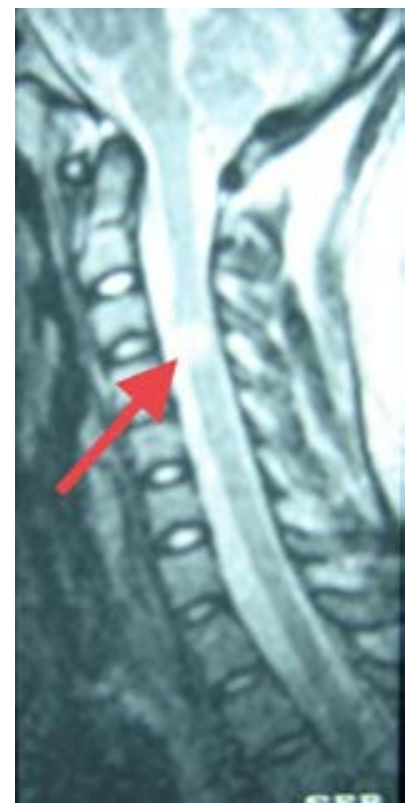

procurando combatir la espasticidad, fatiga, dolor, disfunción vesical, ataxia y los temblores.

El tratamiento durante el evento agudo depende de la gravedad del cuadro; los casos leves pueden remitir espontáneamente. En los cuadros graves o persistentes se recomiendan corticosteroides a dosis altas; la metilprednisolona es el de elección, a dosis de 20 a 30 $\mathrm{mg} / \mathrm{kg} /$ día en pacientes con peso hasta de $30 \mathrm{~kg}$. Con mayor peso, la dosis es de $1 \mathrm{~g} /$ día durante tres a cinco días. El tratamiento debe continuar con prednisona, a dosis de $1 \mathrm{mg} / \mathrm{kg} /$ día, de dos a cuatro semanas, sobre todo en casos que no remita la sintomatología. Cuando no hay repuesta apropiada a esteroides, se utiliza inmunoglobulina intravenosa ( $1 \mathrm{~g} / \mathrm{kg} /$ día, en dos dosis). En pacientes con encefalopatía grave y tetraplejía se recomienda usar plasmaféresis; con lo cual se ha documentado mejoría hasta en el $42 \%$ de los pacientes (nivel de evidencia I). ${ }^{18}$

El pronóstico en general no es bueno, ya que la mayoría de estos pacientes tendrán nuevos episodios o recaídas (0.38 a $1.2 \%$ por año), como ocurrió en las pacientes que describimos en este reporte. En la edad pediátrica, el cuadro clínico es menos grave que en adultos. Una presentación progresiva con síntomas motores y cerebelosos permanentes, los brotes frecuentes en los menores de seis años y el sexo masculino son factores considerados de mal pronóstico. ${ }^{18}$

Tabla 2: Diagnóstico diferencial de esclerosis múltiple.

\section{Tipo de enfermedades}

Genéticas

Infecciosas

Inflamatorias

Metabólicas

Neoplásicas

Estructurales

Vasculares

Intoxicaciones

Misceláneas
Malformación cerebrovascular, vasculopatía cerebrovascular hereditaria, paraparesia espástica hereditaria, degeneración espinocerebelosa, enfermedad por depósitos lisosomales, enfermedad mitocondrial, deficiencia nutricional, acidemia orgánica, enfermedad de los peroxisomas, enfermedad de Wilson

Infecciones por virus: polio, rubéola, VIH y herpes

Infecciones bacterianas, abarcando Brucella y espiroquetas

Enfermedad de Behcet, enfermedad colágeno-vascular, miastenia gravis, sarcoidosis del sistema nervioso

Deficiencia de vitamina B12, deficiencia de ácido fólico, deficiencia de vitamina $\mathrm{E}$

Linfoma intravascular, cáncer metastásico, síndrome paraneoplásico, tumor cerebral primario

Quiste aracnoideo, aracnoiditis, malformación de Arnold-Chiari, espondilosis o hernia de disco, malformación vascular, siringomielia

Síndrome antifosfolípido, arteriopatía cerebral autonómica dominante con infartos subcorticales y leucoencefalopatía, enfermedad de Eales, enfermedad cerebrovascular, vasculopatía retrococlear de Susa, migraña, vasculitis

Óxido nitroso, mielinólisis pontina central, leucoencefalopatía postquimioterapia, neuropatía por radiación, toxicidad por clioquinol con mielopatía subaguda y neuritis óptica, tricloroetileno

Síndrome de fatiga crónica, leucoencefalopatía con desvanecimiento de materia blanca, neuritis sensitiva migratoria, neurorretinitis, neuropatía periférica, desorden de los plexos braquial o lumbosacro, histiocitosis sistémica 


\section{REFERENCIAS}

1. Camacho-Aguilera J. Charcot y su legado a la medicina. Gaceta Médica de México. 2012; 148: 321-326.

2. Domínguez-Moreno R, Morales-Esponda M, Rossiere-Echazarreta $\mathrm{N}$, Olan-Triano R, Gutiérrez-Morales J. Esclerosis múltiple: revisión de la literatura. Revista de la Facultad de Medicina de la UNAM. 2012; 55: 26-35.

3. Polman C, Reingold S, Banwell B, Clanet M, Cohen J, Filippi M et al. Diagnostic criteria for multiple sclerosis: 2010 revisions to the McDonald criteria. Ann Neurol. 2011; 69: 292-302.

4. Duquette P, Murray J, Pleines J, Ebers C. Multiple sclerosis in childhood: clinical profile in 125 patients. J Pediatr. 1987; 11: 359-363.

5. Facchini SA, Harding SA, Waldron II RL. Human immunodeficiency virus-1 infection and multiple sclerosis-like illness in a child. Pediatr Neurol. 2002; 26: 231-235

6. Mirallave PA, Hernández PM, Arnau SA, Hadgigeorgiu I, Sosa HM. El rol de la vitamina D en la esclerosis múltiple. Revista Española de Esclerosis Múltiple. 2012; 5-11.

7. Thompson A, Banwell B, Barkhof F, Carroll W, Coetzee T, Comi $G$ et al. Diagnosis of multiple sclerosis: 2,017 revisions of the McDonald criteria. Lancet Neurol. 2018; 17(2): 162-173.

8. Martinez-Altarriba M, Ramos-Campoy O, Luna-Calcaño I, ArrietaAntón E. Revisión de la esclerosis múltiple (2). Diagnóstico y tratamiento. Medicina de Familia-SEMERGEN. 2015; 41(6):324-328.

9. Robinson-Agramonte M, Guzmeli V, Martínez-Benítez M, InfanteVelázquez E, Galvizo-Sánchez R, Ochoa-Zaldívar M et al. Contribución de la detección de bandas oligoclonales (BOC) en LCR para la confirmación del diagnóstico en esclerosis múltiple. Rev Mex Neuroci. 2005; 6(1): 8-15.

10. Pohl D, Rostasy K, Reiber A, Hanefeld F. CFS characteristic in early-onset multiple sclerosis. Neurology. 2004; 63: 1966-1967.
11. Visudhiphan P, Chiemchanya S, Santadusit S. Optic neuritis in children: Recurrence and subsequent development of multiple sclerosis. Pediatr Neurol. 1995; 13: 293-295.

12. Jones CT. Childhood autoimmune neurologic diseases of the central nervous system. Neurol Clin North Am. 2003; 21: 745-764.

13. García-Merino A, Ara-Callizo A, Fernández-Fernández $O$, LandetePascual L, Moral-Torres E, Rodríguez Antigüedad-Zarrantz A. Consenso para el tratamiento de la esclerosis múltiple 2016. Sociedad Española de Neurología. Neurología. 2017; 32(2): 113119.

14. Aguilar-Juárez P, Castillo-Lara R, Ceballos-Godina M, ColoradoOchoa H, Espinosa-Zacarías P, Flores-Ramírez F. Consenso para el diagnóstico y tratamiento de la esclerosis múltiple en pacientes del ISSSTE. Med Int Méx. 2019; 35(5): 732-771.

15. Lyncet-Mejorado D, Barragán-Pérez E. Esclerosis múltiple en pediatría. Bol Med Hosp Infant Mex. 2006; 63: 40-46.

16. Handel A, Williamson A, Disanto G, Handunnetthi L, Giovannoni G, Ramagopalan S. An updated metaanalysis of risk of multiple sclerosis following infectious mononucleosis. PLoS One. 2010; 5 : 1-5.

17. Filippi M, Preziosa P, Meani A, Ciccarelli O, Mesaros S, Rovira A et al. Prediction of a multiple sclerosis diagnosis in patients with clinically isolated syndrome using the 2016 MAGNIMS and 2010 McDonald criteria: a retrospective study. Lancet Neurol. 2018; 17(2): 133-142.

18. Brenton N, Banwell B Therapeutic approach to the management of pediatric demyelinating disease: multiple sclerosis and acute disseminated encephalomyelitis. Neurotherapeutics. 2016; 13: 84-95.

Conflicto de intereses: los autores declaran que no tienen. 